\title{
Anomalous Anisotropic Diffusion Dynamics of Hydration Water at Lipid Membranes
}

\author{
Yann von Hansen, ${ }^{1,2}$ Stephan Gekle, ${ }^{2,3}$ and Roland R. Netz ${ }^{1,2, *}$ \\ ${ }^{1}$ Department of Physics, Freie Universität Berlin, 14195 Berlin, Germany \\ ${ }^{2}$ Physics Department, Technische Universität München, 85748 Garching, Germany \\ ${ }^{3}$ Physikalisches Institut, Universität Bayreuth, 95440 Bayreuth, Germany \\ (Received 25 March 2013; published 11 September 2013)
}

\begin{abstract}
The diffusional water dynamics in the hydration layer of a dipalmitoylphosphatidylcholine bilayer is studied using molecular dynamics simulations. By mapping the perpendicular water motion on the ordinary diffusion equation, we disentangle free energetic and friction effects and show that perpendicular diffusion is strongly reduced. The lateral water motion exhibits anomalous diffusion up to several nanoseconds and is characterized by even further decreased diffusion coefficients, which by comparison with coarse-grained simulations are explained by the transient corrugated effective free energy landscape imposed by the lipids. This is in contrast to homogenous surfaces, where boundary hydrodynamic theory quantitatively predicts the anisotropy of water diffusion.
\end{abstract}

PACS numbers: 87.16.dj, 02.50.Fz, 05.40.-a, 87.10.Mn

Lipid bilayers are fundamental building blocks of all living matter [1]. Most membrane physiological functions involve transport of molecules within the membrane plane or between the membrane and the bulk compartments [2]. In all these situations, the dynamics of the membrane hydration water is believed to be important [3] and has been experimentally studied by radioactive label [4], NMR [5], inelastic neutron scattering [6], fluorescence spectroscopy [7], IR adsorption [8], and THz spectroscopy techniques [9]. Experimentally, the long-time dynamics of molecules in bacterial cells [10] and crowded fluids [11] as well as transmembrane-protein motion [11] have been found to deviate strongly from the ordinary diffusion law, which was suggested to be of physiological relevance in preventing prospective reaction partners to prematurely run away from each other [10]. In atomistic simulations, lateral lipid diffusion was found to be anomalous over extended intermediate time scales for different compositions [12], to exhibit cooperative motion [13], and to reflect internal degrees of freedom [14]. Transmembrane proteins diffuse even slower and show enhanced anomalous diffusion behavior [15]. Membrane hydration water dynamics was considered in a few theoretical works: Water diffusion perpendicular to the membrane plane was simulated in order to predict transmembrane water transport [16] and water dynamics as relevant to NMR experiments [17]. Water diffusion a fixed distance away from the bilayer surface was extracted from molecular dynamics (MD) simulations [18], but the full tensorial anisotropy of the hydration water diffusivity was not considered.

In this paper, we examine the dynamics of individual water molecules in the vicinity of a dipalmitoylphosphatidylcholine (DPPC) bilayer based on the trajectory analysis of extensive MD simulations. Once the free energy profile perpendicular to the membrane plane is accounted for in the stochastic analysis, water diffusion is found to be strongly reduced next to the membrane. We reveal anisotropic diffusion behavior of the hydration water that markedly differs from the one at solid surfaces, where the water diffusion is also substantially slowed down but in quantitative agreement with continuum hydrodynamic theory. As we show by coarse-grained simulations, this effect can be traced back to the transient but long-lived corrugated free energy landscape imposed by the lipids. The lateral water dynamics consequently shows the characteristics of obstructed diffusion, similar to the dynamics in crowded fluids [11]. We expect the diffusion of other solutes at membranes to exhibit similar features and thus our findings to be relevant for a broad range of physiological processes ranging from intercellular signaling to drug delivery.

Our simulation setup resembles that of previous studies [19-21] and consists of a lipid bilayer formed by 72 DPPC molecules and 2278 SPC/E water molecules; see the simulation snapshot in Fig. 1(a). Production runs have a total length of $\approx 6 \mu \mathrm{s}$ at a temperature of $T=320 \mathrm{~K}$ in the $N V T$ ensemble with a mean area of $\approx 0.65 \mathrm{~nm}^{2}$ per DPPC molecule; further details on the simulations are found in
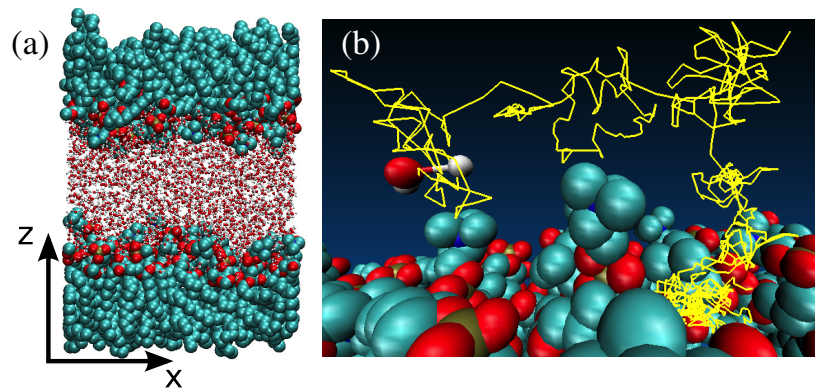

FIG. 1 (color online). (a) Snapshot of the simulation box. (b) Trajectory of a water molecule in the vicinity of the membrane: the path of 100 ps duration starts in the lower right. 
the Supplemental Material (SM) [22]. We note that finitesize effects for the dynamics of individual lipids have previously been observed in similar systems [23] but are not expected to have a major influence on our results; see the SM for details [22].

Water motion parallel to the membrane surface is characterized by the mean square displacement (MSD)

$$
\left\langle[\Delta x(t)]^{2}\right\rangle_{z_{0}} \equiv\left\langle\left[x\left(t^{\prime}+t\right)-x\left(t^{\prime}\right)\right]^{2}\right\rangle_{z_{0}}
$$

of the $x$ coordinate of the water oxygen (and similarly for the $y$ coordinate). In Eq. (1), $\langle\ldots\rangle_{z_{0}}$ denotes the double average over the reference time $t^{\prime}$ and all water molecules, for which $\left|z\left(t^{\prime}+\tau\right)-z_{0}\right| \leq 0.25 \mathrm{~nm}$ is fulfilled for the entire time span $0 \leq \tau \leq t$; i.e., only trajectories which remain within an interval of $0.5 \mathrm{~nm}$ centered around a prescribed distance $z_{0}$ from the membrane midplane are considered. In order to avoid artifacts due to the diffusion of the membrane as a whole, we have subtracted the lateral center-of-mass position of the nearest membrane leaflet from the water coordinates [24,25].

Lateral water MSDs for different distances $z_{0}$ from the membrane center (denoted by different colors) are shown as dashed lines in Fig. 2(a); the perfect agreement for $x$ and $y$ testifies to the statistical data quality. While the subpicosecond dynamics is independent of $z_{0}$, the MSDs differ considerably on larger time scales [18]: whereas water molecules in the center of the water slab $\left(z_{0}=3.5 \mathrm{~nm}\right)$ show MSD curves that are indistinguishable from bulk water [26] (thick gray line), closer to the membrane, the water MSD curves approach the lateral MSDs of the lipids' phosphorus atoms (black dots).

Water MSD curves become statistically unreliable for $t \gtrsim 100 \mathrm{ps}$ because very few water trajectories stay within a given interval around $z_{0}$ for such long times. Moreover, the trajectory ensemble changes with time, leading to an artificial decrease of the MSDs in Fig. 2(a), as is discussed in Ref. [27] and in the SM [22]. To reliably determine lateral MSDs on the nanosecond time scale, we resort to simulations where $\sim 6 \%$ of all water molecules are harmonically constrained in the $z$ direction. The resulting MSDs are shown as solid lines in Fig. 2 and compare well to the unconstrained MSDs on times up to $\sim 20 \mathrm{ps}$.

The local slopes of the MSD data in the doublelogarithmic representation correspond to the timedependent exponent $\alpha$, which is shown in the inset of Fig. 2(a): For bulklike water (blue curves), we observe a smooth transition from superdiffusive behavior $\alpha>1$ for times $t \lesssim 200$ fs to a subdiffusive regime with $\alpha \approx 0.9$ on the picosecond time scale, and a subsequent crossover to the exponent $\alpha=1$ associated with normal diffusion for $t \gtrsim 10 \mathrm{ps}$. When approaching the membrane, the anomalous diffusion regime extends to time scales of up to nanoseconds and the exponents $\alpha$ decrease. For water molecules in direct proximity of the membrane $\left(z_{0}=1.5 \mathrm{~nm}\right)$, typical MSD exponents are $\approx 0.4$, similar

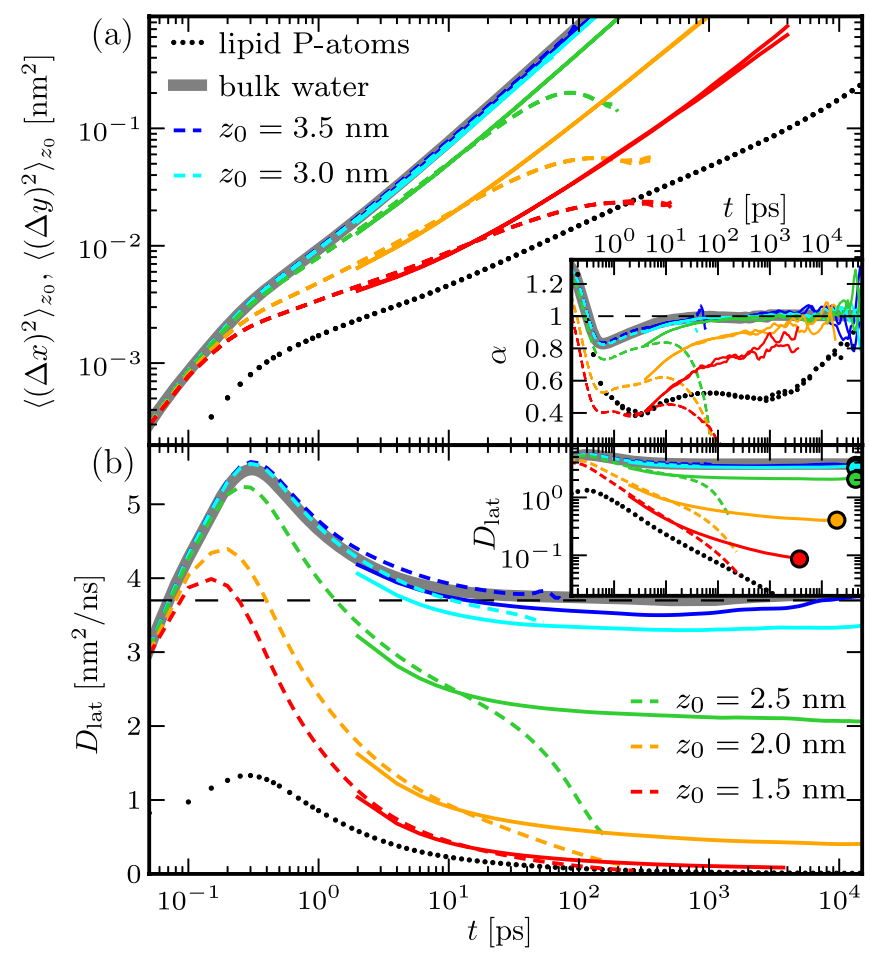

FIG. 2 (color online). (a) Lateral MSDs [Eq. (1)] for water at different distances $z_{0}$ from the membrane center (colored lines), for lipid heads (black dots), and for bulk water [26] (gray lines, hidden under the $z_{0}=3.5 \mathrm{~nm}$ water data). Corresponding local exponents $\alpha$ are shown in the inset. (b) Resulting timedependent diffusion coefficients $D_{\text {lat }}$ [Eq. (2)], shown on a double-logarithmic scale in the inset. Filled colored circles denote upper bounds for the long-time diffusion coefficients. In all panels and insets, the same color coding is used, and data for unconstrained and harmonically trapped water molecules are displayed as dashed and solid lines, respectively.

to those of the lipid head groups themselves [12,14], demonstrating a tight coupling of water and lipid motion further analyzed below.

Time-dependent lateral diffusion coefficients

$$
D_{\text {lat }}\left(t ; z_{0}\right) \equiv\left\langle[\Delta x(t)]^{2}+[\Delta y(t)]^{2}\right\rangle_{z_{0}} /(4 t)
$$

are shown in Fig. 2(b) using the same color coding as in Fig. 2(a). While the ballistic regime $\Delta x^{2}=\Delta y^{2} \propto t^{2}$ at short times gives rise to a linearly increasing diffusion coefficient, a maximum in the subpicosecond range and slow subsequent saturation are observed. The diffusion coefficients in the membrane vicinity continue to decrease even beyond several nanoseconds, as is best seen in the double-logarithmic representation of the data in the inset of Fig. 2(b), where upper bounds for the long-time diffusion coefficients are indicated by colored circles.

We next turn to water diffusion perpendicular to the membrane surface. Several methods to extract diffusivity profiles in inhomogenous systems exist $[16,27,28]$; here, we employ a mean first-passage time (MFPT) analysis to disentangle contributions of free energy and diffusivity to 
the water kinetics $[29,30]$. For the MFPT $\tau_{\mathrm{fp}}\left(z, z_{t}\right)$ to first reach a target distance $z_{t}$ given a start at $z$, an exact solution of the one-dimensional diffusion equation in terms of the free energy $F(z)$ and diffusivity profiles $D_{z}(z)$ exists [31,32], which can be inverted and allows us to determine $D_{z}(z)$ from measured MFPTs via

$$
D_{z}(z)=-\frac{e^{F(z) /\left(k_{B} T\right)}}{\partial \tau_{\mathrm{fp}}\left(z, z_{t}\right) / \partial z} \int_{z_{\text {refl }}}^{z} \mathrm{~d} z^{\prime} e^{-F\left(z^{\prime}\right) /\left(k_{B} T\right)} .
$$

Here, we impose a reflective boundary at $z_{\text {refl }}=0.475 \mathrm{~nm}$ where the free energy has a value of $\approx 12 k_{B} T$. Water density $\rho(z)$, the resulting free energy profile $F(z) \equiv$ $-k_{B} T \log \left[\rho(z) / \rho_{0}\right]$, and the Gibbs dividing surface (GDS) are shown in Fig. 3(a). MFPTs for different target distances $z_{t}$ as extracted from MD trajectories are presented in Fig. 3(b). Diffusivity profiles resulting from Eq. (3) are denoted by solid lines in Fig. 3(c) using the same color coding as in Fig. 3(b). The diffusivity profile reveals bulklike dynamics with a diffusion coefficient $D_{\mathrm{H}_{2} \mathrm{O}} \approx 3.7 \mathrm{~nm}^{2} / \mathrm{ns}$ at $T=320 \mathrm{~K}$ [26] [indicated by a horizontal dashed line in Fig. 3(c)] in a small region in the center of the water slab only. When approaching the membrane, the diffusivity smoothly decreases and levels off at values $\sim 0.2 \mathrm{~nm}^{2} / \mathrm{ns}$ in the lipid head group region, about a factor of 20 smaller than in bulk.

We observe that diffusivity profiles from MFPT curves for different target distances $z_{t}$ superimpose well except for $z_{t}-z \lesssim 0.25 \mathrm{~nm}=\delta z$. These deviations are caused by non-Markovian effects on time scales $(\delta z)^{2} / D_{z}$ ranging from $\sim 10 \mathrm{ps}$ in the bulklike region of the water slab to $\sim 300$ ps near the lipids. The overlap of the diffusivity profiles demonstrates that perpendicular diffusion on larger length and longer time scales is Markovian and accurately described by the diffusion equation, including the free energy profile.

For comparison, we also indicate the upper bounds for the lateral diffusivity $D_{\text {lat }}(z)$ from the inset of Fig. 2(b) as open circles in Fig. 3(c). While both perpendicular and parallel diffusion are slowed down in a region with a thickness of $2 \mathrm{~nm}$ close to the membrane surface, a significant anisotropy $D_{\text {lat }}(z) / D_{z}(z)<1$ is observed in the inset of Fig. 3(c) (filled circles), e.g., $D_{\text {lat }} \approx D_{z} / 3$ for $z_{0}=1.5 \mathrm{~nm}$.

As we show next, this observation is in marked contrast to the water dynamics at solid homogenous interfaces: In Fig. 4, we reproduce water free energy and diffusivity profiles at $\mathrm{H}$ - (hydrophobic) and $\mathrm{OH}$ - (hydrophilic) terminated diamond surfaces obtained using similar methods as described above [30]. The bulk diffusion coefficient $D_{\mathrm{H}_{2} \mathrm{O}} \approx 2.7 \mathrm{~nm}^{2} / \mathrm{ns}$ at $T=300 \mathrm{~K}[26]$ is indicated as horizontal dashed lines in Figs. 4(c) and 4(d). Exactly opposite from the behavior at the lipid bilayer, the lateral diffusivity (blue squares) is larger than the perpendicular one (red circles) for both surface polarities, as expected based on continuum hydrodynamics: The colored lines show

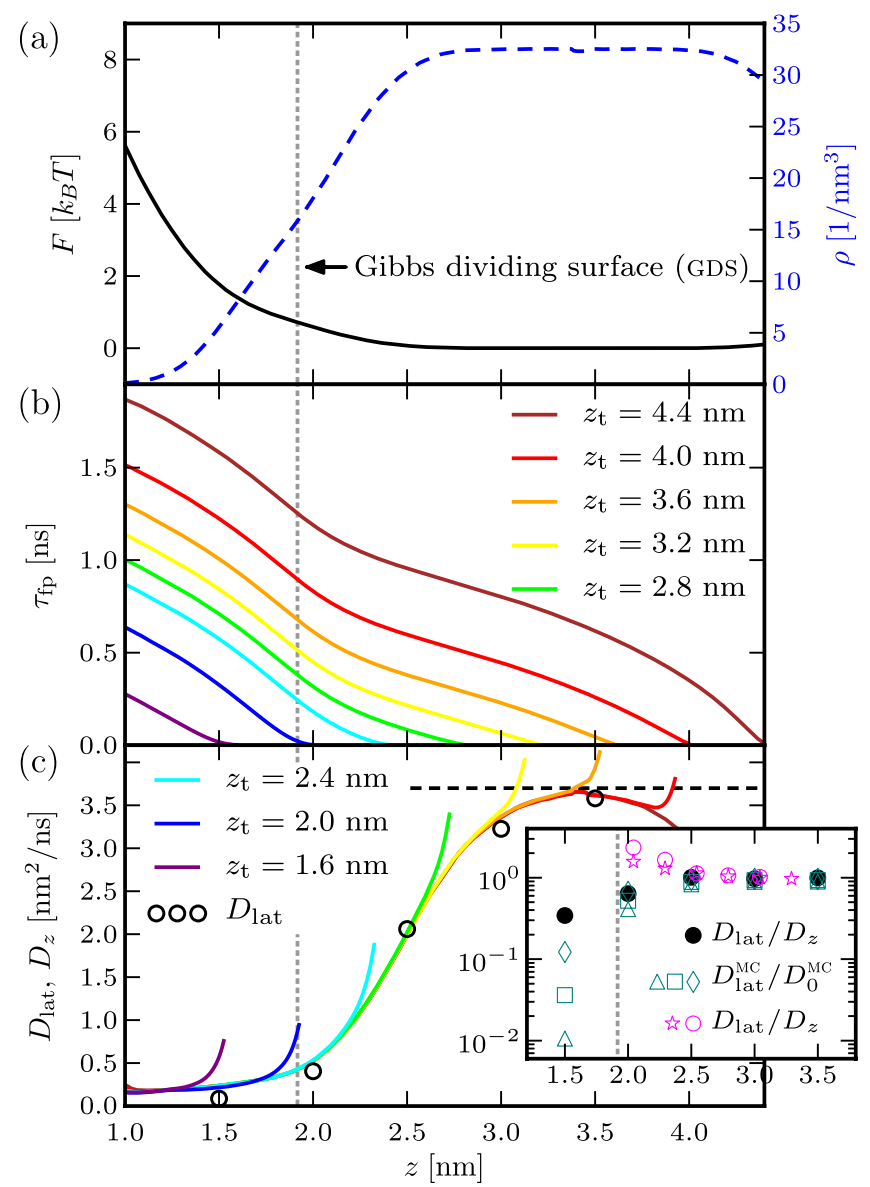

FIG. 3 (color online). (a) Water density $\rho(z)$ and free energy profile $F(z)$. (b) Water MFPTs to first reach a target distance $z_{t}$ when starting off from $z$. (c) Perpendicular water diffusivity profiles $D_{z}(z)$ (solid colored lines) derived via Eq. (3). Open circles denote the long-time lateral diffusivities from the inset of Fig. 2(b). Inset: Water diffusion anisotropy $D_{\text {lat }} / D_{z}$ at the membrane (filled circles), at solid homogenous hydrophobic (stars) and hydrophilic (open circles) surfaces (Fig. 4), and the lateral rescaled diffusion constant $D_{\text {lat }}^{\mathrm{MC}} / D_{0}^{\mathrm{MC}}$ (open triangles, squares, and diamonds) from MC simulations (Fig. 5); the $z$ coordinates of the solid homogenous surfaces have been chosen such that the positions of the respective GDSs agree.

predictions for the anisotropic mobility of a sphere with a radius $R_{\text {hyd }}$ adjusted to match the bulk diffusivity $D_{\mathrm{H}_{2} \mathrm{O}}$ via the Stokes relation $R_{\text {hyd }} \equiv k_{B} T /\left(6 \pi \eta_{0} D_{\mathrm{H}_{2} \mathrm{O}}\right) \approx 0.11 \mathrm{~nm}$ using the SPC/E shear viscosity $\eta_{0} \approx 0.71 \mathrm{mPa} \cdot \mathrm{s}$ [33]. For the hydrophilic $\mathrm{OH}$-terminated surface, we independently estimate the stagnation layer position as $z_{\text {stag }}=$ $2.19 \mathrm{~nm}$ [33] and in Fig. 4(d) show the parallel or perpendicular sphere mobilities at a no-slip surface located at $z_{\text {stag }}-R_{\text {hyd }} \approx 2.08 \mathrm{~nm}$ (colored lines; see the SM for details) $[22,34,35]$. The hydrophobic H-terminated diamond surface in MD simulations exhibits a slip length $b \approx 2.5 \mathrm{~nm}$ [33]; in Fig. 4(c), we show analytic predictions for the anisotropic sphere mobilities, including this finite surface slip [36]. Apart from oscillations in the mobilities 


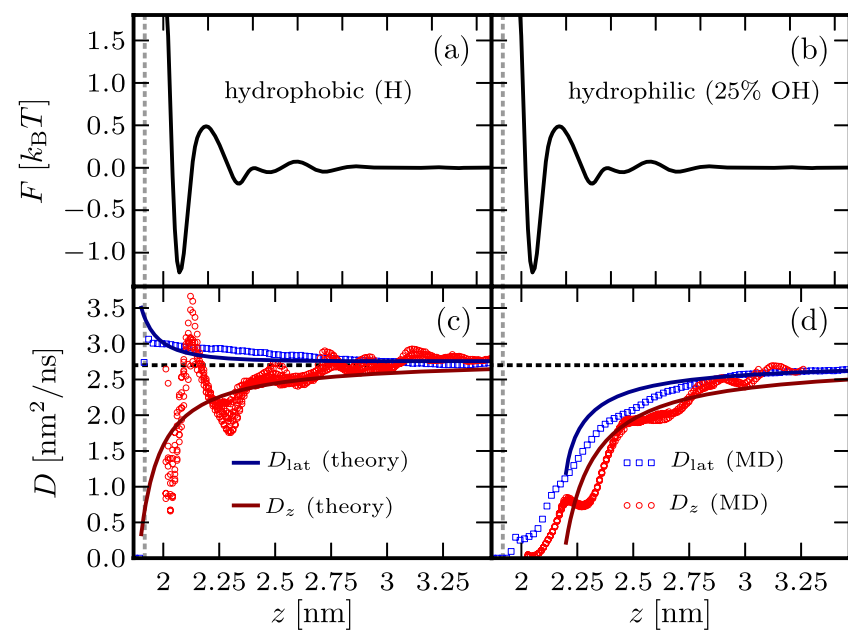

FIG. 4 (color online). (a), (b) Water free energy profiles at hydrophobic or hydrophilic solid surfaces from MD simulations [30]. (c), (d) Diffusivity profiles for motion parallel and perpendicular to the surfaces from MD simulations (symbols) [30] and estimates from hydrodynamic theory (lines). The dotted vertical lines denote the positions of the GDSs.

due to molecular interfacial layering, the continuum theory is in excellent agreement with the MD data, which is particularly meaningful since no adjustable fit parameter is introduced; i.e., slip and stagnation length are independently determined $[22,33]$. Both continuum hydrodynamics and MD simulations show that the lateral diffusivity at solid surfaces is larger than the perpendicular one, in contrast to the membrane scenario. This is highlighted in the inset of Fig. 3(c), where we compare the ratio $D_{\text {lat }} / D_{z}$ for hydrophobic and hydrophilic solid surfaces (magenta stars and open circles, results have been averaged over $0.25 \mathrm{~nm}$ ) with the membrane results (filled black circles).

To understand the discrepancy between water diffusion in the lipid interfacial region, where $D_{\text {lat }} / D_{z}<1$, and next to solid surfaces, where $D_{\text {lat }} / D_{z}>1$, we examine the influence of the lateral bilayer structure. For this, we calculate lateral water density profiles $\bar{\rho}_{z_{0}, \tau}(x, y)$ that are averaged over a time $\tau$ for different distances $z_{0}$ from the membrane. A density profile for $z_{0}=2 \mathrm{~nm}$ and $\tau=50 \mathrm{ps}$ is shown in Fig. 5; other examples are found in the SM [22]. A transient heterogenous and strongly structured water distribution is revealed, which averages out on time scales of nanoseconds only. To estimate the effect on lateral long-time water diffusion, we perform Monte Carlo (MC) simulations in the corresponding free energy landscape $\bar{F}_{z_{0}, \tau}(x, y) \equiv-k_{B} T \log \left[\bar{\rho}_{z_{0}, \tau}(x, y) / \rho_{0}\right]$, which is laterally discretized with lattice constant $\delta x \approx \delta y[22]$. For illustration, a 500 step MC trajectory is superimposed on the density profile as a black line in Fig. 5. The resulting lateral MSD (red line in the inset of Fig. 5) shows a pronounced subdiffusive regime and a decreased long-time diffusion coefficient-both characteristic features for obstructed diffusion in rough

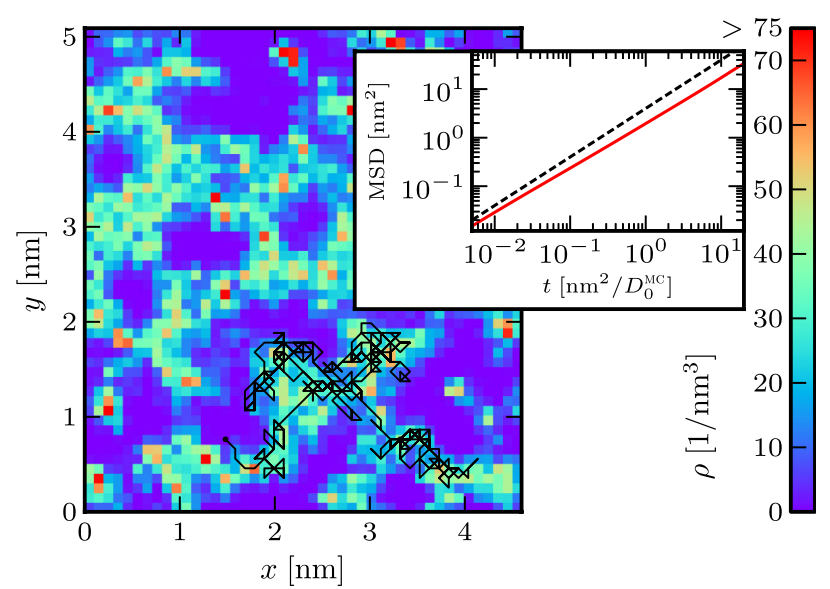

FIG. 5 (color online). Water density profile $\bar{\rho}_{z_{0}, \tau}(x, y)$ averaged in a slab $\left|z-z_{0}\right| \leq 0.25 \mathrm{~nm}$ centered at $z_{0}=2 \mathrm{~nm}$ over a time window $\tau=50$ ps with lateral discretization $\delta x \approx \delta y \approx 0.1 \mathrm{~nm}$. A sample MC trajectory of 500 steps is shown as thin black line. The inset shows the lateral MSD from MC simulations (solid red line) and the reference MSD in an unstructured environment (dotted black line).

environments [11]. The long-time diffusion critically depends on the averaging time $\tau$, less so on the spatial discretization: Open symbols in the inset of Fig. 3(c) denote the ratio $D_{\text {lat }}^{\mathrm{MC}} / D_{0}^{\mathrm{MC}}$ for $\tau=50 \mathrm{ps}$ (triangles), $\tau=100 \mathrm{ps}$ (squares), and $\tau=250 \mathrm{ps}$ (diamonds), where $D_{0}^{\mathrm{MC}} \equiv(\delta x)^{2} /(2 \delta t)$ is the bare MC diffusion coefficient and $\delta t$ the MC time step. As expected, the free energy landscape slows down the lateral water motion considerably: the shorter the averaging time, the more corrugated the potential, and thus the slower the diffusion (the overall match of water dynamics in the MD and MC simulations would obviously require time-dependent free energy landscapes). We see that the water diffusion anisotropy from the MC simulation, which comprises the obstructing free energy landscape due to the inhomogenous lipid distribution but no hydrodynamic effects, is more pronounced than the anisotropy extracted from the membrane simulations (filled circles)

We conclude that the observed diffusion anisotropy of membrane hydration water results from the interplay of two competing effects: (i) Continuum hydrodynamics alone speeds up the lateral diffusion compared to the perpendicular one, regardless of the surface polarity, as confirmed by our MD simulations at solid homogenous surfaces, implying $D_{\text {lat }} / D_{z}>1$, while (ii) the lateral diffusion dynamics is considerably slowed down by the heterogenous transient but long-lived free energy landscape imposed by the lipids, implying $D_{\text {lat }} / D_{z}<1$, as shown by our coarse-grained MC simulations without hydrodynamics. As seen in the inset of Fig. 3(c), both effects roughly compensate for $z \gtrsim 2.5 \mathrm{~nm}$, yielding almost isotropic diffusive properties, while the second effect dominates for $z \lessgtr 2.5 \mathrm{~nm}$, giving rise to a pronounced anisotropy with $D_{\text {lat }} / D_{z}<1$. 
Our findings imply that efficient transport parallel to the membrane surface necessarily also involves fluctuations perpendicular to the lipid leaflet, as shown in Fig. 1(b). In turn, attraction towards the lipid bilayer would constitute a simple mechanism for significantly suppressing lateral diffusive motion. We also expect our results to be relevant for the diffusion of solutes at the surfaces of proteins or other structured biological surfaces and to have implications for surface binding and reaction kinetics [37].

The authors acknowledge the Leibniz Rechenzentrum (LRZ) Munich and the Forschungszentrum Jülich for supercomputing access, financial support from the DFG within SFB 1078 and under Project No. GE2214, as well as from the Elitenetzwerk Bayern in the framework of CompInt and from the Volkswagen Foundation.

*rnetz@physik.fu-berlin.de

[1] G. Cevc and D. Marsh, Phospholipid Bilayers-Physical Principles and Models (Wiley, New York, 1987).

[2] O. G. Mouritsen, Life-As a Matter of Fat (Springer, New York, 2005).

[3] S. K. Pal and A. H. Zewail, Chem. Rev. 104, 2099 (2004).

[4] J. L. Rigaud, Y. Lange, and C. M. Garybobo, Biochim. Biophys. Acta 266, 72 (1972).

[5] S. Konig, E. Sackmann, D. Richter, R. Zorn, C. Carlile, and T. M. Bayerl, J. Chem. Phys. 100, 3307 (1994).

[6] J. Swenson, F. Kargl, P. Berntsen, and C. Svanberg, J. Chem. Phys. 129, 045101 (2008).

[7] S. K. Pal, D. Sukul, D. Mandal, and K. Bhattacharyya, J. Phys. Chem. B 104, 4529 (2000).

[8] W. Zhao, D. E. Moilanen, E. E. Fenn, and M. D. Fayer, J. Am. Chem. Soc. 130, 13927 (2008).

[9] M. Hishida and K. Tanaka, Phys. Rev. Lett. 106, 158102 (2011).

[10] I. Golding and E. C. Cox, Phys. Rev. Lett. 96, 098102 (2006).

[11] J. Szymanski and M. Weiss, Phys. Rev. Lett. 103, 038102 (2009); N. Malchus and M. Weiss, Biophys. J. 99, 1321 (2010).

[12] J.-H. Jeon, H. M.-S. Monne, M. Javanainen, and R. Metzler, Phys. Rev. Lett. 109, 188103 (2012).

[13] E. Falck, T. Róg, M. Karttunen, and I. Vattulainen, J. Am. Chem. Soc. 130, 44 (2008).

[14] E. Flenner, J. Das, M.C. Rheinstädter, and I. Kosztin, Phys. Rev. E 79, 011907 (2009).

[15] M. Javanainen, H. Hammaren, L. Monticelli, J. H. Jeon, M. S. Miettinen, H. Martinez-Seara, R. Metzler, and I. Vattulainen, Faraday Discuss. 161, 397 (2013).
[16] S. J. Marrink and H. J. C. Berendsen, J. Phys. Chem. 98, 4155 (1994); 100, 16729 (1996).

[17] K. Åman, E. Lindahl, O. Edholm, P. Håkansson, and P. O. Westlund, Biophys. J. 84, 102 (2003).

[18] F. Y. Hansen, G. H. Peters, H. Taub, and A. Miskowiec, J. Chem. Phys. 137, 204910 (2012).

[19] H. A. Stern and S.E. Feller, J. Chem. Phys. 118, 3401 (2003).

[20] C. F. Lopez, S. O. Nielsen, M. L. Klein, and P. B. Moore, J. Phys. Chem. B 108, 6603 (2004).

[21] E. Schneck, F. Sedlmeier, and R. R. Netz, Proc. Natl. Acad. Sci. U.S.A. 109, 14405 (2012).

[22] See Supplemental Material at http://link.aps.org/ supplemental/10.1103/PhysRevLett.111.118103 for details concerning the simulation protocol, the data analysis, the MC simulations, and the comparison of diffusivity profiles at homogenous solid surfaces to estimates from hydrodynamic theory.

[23] A. H. de Vries, I. Chandrasekhar, W. F. van Gunsteren, and P. H. Hünenberger, J. Phys. Chem. B 109, 11643 (2005); F. Castro-Román, R. W. Benz, S. H. White, and D. J. Tobias, J. Phys. Chem. B 110, 24157 (2006); J. B. Klauda, B. R. Brooks, and R. W. Pastor, J. Chem. Phys. 125, 144710 (2006).

[24] E. Lindahl and O. Edholm, J. Chem. Phys. 115, 4938 (2001).

[25] J. P. M. Jämbeck and A. P. Lyubartsev, J. Phys. Chem. B 116, 3164 (2012).

[26] Y. von Hansen, F. Sedlmeier, M. Hinczewski, and R. R. Netz, Phys. Rev. E 84, 051501 (2011).

[27] P. Liu, E. Harder, and B. J. Berne, J. Phys. Chem. B 108, 6595 (2004).

[28] J. Mittal, T. M. Truskett, J. R. Errington, and G. Hummer, Phys. Rev. Lett. 100, 145901 (2008).

[29] M. Hinczewski, Y. von Hansen, J. Dzubiella, and R. R. Netz, J. Chem. Phys. 132, 245103 (2010).

[30] F. Sedlmeier, Y. von Hansen, L. Mengyu, D. Horinek, and R. R. Netz, J. Stat. Phys. 145, 240 (2011).

[31] G. H. Weiss, Adv. Chem. Phys. 13, 1 (2009).

[32] C. W. Gardiner, Handbook of Stochastic Methods for Physics, Chemistry and the Natural Sciences, Springer Series in Synergetics Vol. 13 (Springer, New York, 2002).

[33] C. Sendner, D. Horinek, L. Bocquet, and R. R. Netz, Langmuir 25, 10768 (2009).

[34] G. S. Perkins and R. B. Jones, Physica (Amsterdam) A 189, 447 (1992).

[35] M. Stimson and G. B. Jeffery, Proc. R. Soc. A 111, 110 (1926).

[36] E. Lauga and T. M. Squires, Phys. Fluids 17, 103102 (2005).

[37] M. A. Lomholt, I. M. Zaid, and R. Metzler, Phys. Rev. Lett. 98, 200603 (2007). 